\title{
Modelización global del proceso de colada continua
}

\author{
J. Barco*, J. Palacios*, C. Ojeda*y M. Ojanguren*
}

\begin{abstract}
Resumen En el proceso de colada continua contribuyen complicados fenómenos físicos con un marcado efecto en la calidad final del producto y en la productividad del proceso. Además, los distintos fenómenos físicos no aparecen de forma aislada, sino que interactúan unos con otros de manera acoplada, afectando a la calidad del semiproducto. El correcto diseño y ajuste es, por lo tanto, una tarea que requiere un gran conocimiento tanto de los distintos fenómenos que intervienen como de sus interacciones. En este trabajo se presenta un novedoso método de simulación que resuelve el proceso en su conjunto, siendo las variables del proceso las únicas condiciones de contorno. Mediante la utilización conjunta de herramientas comerciales (FLUENT y ABAQUS) y una serie de modelos de desarrollo propio se resuelven las interacciones entre los distintos fenómenos.
\end{abstract}

Palabras clave Colada continua. Modelización. Fundentes. Solidificación. Refrigeración.

\section{Global modelling of continuous casting process}

\begin{abstract}
In the continuous casting process the contribution of complicated physical phenomena has an important effect in the final quality of the product and productivity of the process. Moreover, the different physical phenomena do not act in isolation, they interact in a coupled way affecting the quality of the semiproduct. Therefore, a correct design and setting is a work that requires a good knowledge of the different phenomena that are present and their interactions. In this work, a simulation methodology that solves all the process as a whole is presented, where the variables of the process are the only boundary conditions. The interactions between the different phenomena are solved with the use of commercial applications (FLUENT and ABAQUS) and home developed models.
\end{abstract}

Keywords Continuous casting. Modelling. Mould fluxes. Solidification. Refrigeration.

\section{INTRODUCCIÓN}

La simulación numérica viene siendo utilizada por los fabricantes más importantes de maquinaria de colada continua, habiéndose visto incrementada en los últimos años junto con el fuerte desarrollo comercial de software de ingeniería. Sin embargo, la complejidad del fenómeno global obliga a abordar la simulación de una manera parcial, en la que el modelo se centra en alguno de los fenómenos mientras que el resto se asume en función de hipótesis simplificadas. Esta circunstancia no invalida los resultados obtenidos y, de hecho, se han obtenido avances importantes utilizando este tipo de técnicas; además pone de manifiesto las limitaciones del estado del arte de la simulación numérica en este campo.

En este trabajo, se estudian de forma acoplada los distintos fenómenos que intervienen en el pro- ceso de colada continua, tales como la refrigeración, comportamiento de los fundentes, oscilación, sistema de alimentación al molde y la deformación de la capa solidificada que actúan de forma acoplada.

La metodología empleada se resume en el esquema de la figura 1 .

\section{DESCRIPCIÓN DE LOS MODELOS}

\subsection{Modelo CFD}

Se resuelve al mismo tiempo la refrigeración del molde con agua, la solidificación del acero, así como la transferencia de calor a través de los fundentes, mediante una subrutina de usuario. Se ha simulado en 3D la geometría completa de una colada convencional real, incluyendo todos estos aspectos.

\section{$\left(^{*}\right) \quad$ LABEIN - TECNALIA.}




\section{METODOLOGÍA}

Interpolación de los flujos de calor en la interfase molde-palanquilla

\section{Modelo 3D CFD resolviendo: \\ 1. Refrigeración del molde con agua \\ 2. Solidificación \\ 3. Flujo de calor a través de la región de fundentes mediante subrutina de usuario}

Figura 1. Esquema resumen de la metodología seguida.

Figure 1. Schematic summary of the developed methodology.

La subrutina implementada es acoplada con el modelo CFD, tomando la temperatura superficial del acero $\left(T_{s}\right)$ y la temperatura superficial de la cara caliente del molde $\left(T_{m}\right)$, siendo función de punto. El modelo matemático de transferencia de calor se basa en ecuaciones derivadas del balance de masa y energía de los fundentes ${ }^{[1]}$ (se describe brevemente en la sección siguiente), y la variable que se resuelve es el flujo de calor que se impone en el modelo CFD como una condición de contorno en la interfase acero-molde. Por otro lado, el flujo de calor en la zona de los sprays se estima según Brimacombe $^{[2]}$. Se ha hecho uso del modelo de turbulencia RNG (2 ecuaciones). El acero considerado es el 100 Cr6, y las propiedades térmicas se han tomado del software comercial IDS.

\subsection{Modelización del flujo de calor en el hueco entre acero y molde}

\subsubsection{Balance de masa}

Suponiendo que el consumo de fundente, $Q_{f l u x}$ $\left(\mathrm{Kg} / \mathrm{m}^{2}\right)$, es conocido, se impone el siguiente balance de masa para cada sección transversal del hueco existente entre el molde y el acero. Además, se tiene en cuenta que el fundente puede estar en estado sólido y líquido. La profundidad de la marca de oscilación se tiene en cuenta mediante $d_{o s c}$, y los espesores de fundente sólido y líquido son $d_{s}$ y $d_{l}$, respectivamente.

$$
\frac{Q_{\text {fundente }} V_{c}}{\rho_{\text {fundente }}}=V_{s} d_{s}+V_{l} d_{l}+V_{c} d_{o s c}
$$

La capa sólida se supone que se mueve a una velocidad constante $V_{s}$, que es una fracción $f$ (experimental) de la velocidad de colada $V_{c}$. En cuanto a la velocidad media del fundente líquido $V_{l}$, la velocidad media axial se calcula a partir de la ecuación de Navier-Stokes (régimen laminar debido a la alta viscosidad del fundente líquido).

$$
\begin{aligned}
& \frac{\partial \tau_{x z}}{\partial x}=\frac{\partial}{\partial x}\left(\mu \frac{\partial V_{z}}{\partial x}\right)=-\rho_{\text {fundente }} g+\frac{\partial P}{\partial z} \\
& \frac{\partial P}{\partial z}=\rho_{\text {acero }} g
\end{aligned}
$$

$$
V_{l}=\frac{V_{c}+V_{s}(n+1)}{n+2}+\frac{\left(\rho_{\text {acero }}-\rho_{\text {fundente }}\right) g d_{1}^{2}}{\mu\left(T_{s}^{\prime}\right)(n+2)^{2}(n+3)}
$$

siendo $n$ un coeficiente empírico de la viscosidad.

\subsubsection{Balance de energía}

La transferencia de calor, $Q$, a través del hueco entre el molde y el acero, se calcula mediante las siguientes ecuaciones, que tienen en cuenta el flujo de calor por conducción y por radiación (primer y segundo término en la parte derecha de la ecuación $\left.h_{\text {gap }}\right)$ :

$$
Q=h_{g a p}\left(T_{s}-T_{m}\right)
$$

$$
\begin{aligned}
& h_{g a p}=\frac{1}{\frac{d_{a}}{k_{a}}+\frac{d_{s}}{k_{s}}+\frac{d_{l}}{k_{l}}+\frac{d_{\text {eff }}}{k_{\text {eff }}}+h_{\text {mf }}+h_{f s}}+ \\
& +\frac{m^{2} \sigma\left(T_{s}^{2}+T_{\text {crisal }}^{2}\right)\left(T_{s}+T_{\text {cristal }}\right)}{0.75 a\left(d_{l}+d_{\text {eff }}\right)+\frac{1}{e_{s}}+\frac{1}{e_{m}}-1}
\end{aligned}
$$

siendo $k_{i}$, las conductividades correspondientes, $e_{i}$, las emisividades, $m$, el índice de refracción, $a$, el coeficiente de absorción y $T_{\text {cristal }}$ la temperatura de solidificación de los fundentes.

Para completar el sistema de ecuaciones se dispone de otra ecuación que resuelve la solidificación de los fundentes a partir de la ecuación simplificada unidimensional:

$$
\frac{d\left(d_{s}\right)}{d t}\left[\Delta h_{\text {solidificación }}+c_{p}\left(T-T_{\text {cristal }}\right)\right] \rho_{\text {fundente }}=Q_{1}-Q_{2}
$$


donde, $Q_{1}$ y $Q_{2}$ son los flujos de calor extraídos de la superficie del molde e introducidos por el acero en la interfase sólido-liquido de los fundentes, respectivamente. Como el calor latente del fundente es inferior al $3 \%$ del calor transferido a través del hueco entre acero y molde, queda que:

$$
Q_{1}=Q_{2} \Rightarrow \frac{\left(T_{\text {crisal }}-T_{m}\right)}{\frac{d_{s}}{k_{s}}}=\frac{\left(T_{s}-T_{\text {crisal }}\right)}{\frac{d_{l}}{k_{l}}}
$$

\subsubsection{Forma del menisco}

Se tiene en cuenta en la parte superior la forma del menisco, que es dada por la ecuación obtenida por Bikerman y Matijevic para el caso de menisco estático ${ }^{[3]}$.

\subsection{Modelo de elementos finitos}

Mediante una subrutina programada a tal efecto se aplican en el modelo FEM los flujos de calor procedentes del estudio de CFD para el cálculo las tensiones y deformaciones producidas. Por razones de tiempos de computación, inicialmente, se ha aplicado a un modelo 2D transversal sobre $1 / 4 \mathrm{de}$ la sección de la palanquilla.

Para ello, se utiliza una formulación donde se resuelve acopladamente, en transitorio, las ecuaciones de transferencia de calor, la solidificación y las tensiones-deformaciones sobre elementos de deformación plana, teniendo en cuenta, además, la presión ferrostática dependiente de la altura. La hipótesis de deformación plana es más válida a mayores espesores de capa de acero solidificado, ya que inicialmente el acero se contrae libremente y, por tanto, puede deformarse en dirección perpendicular a la sección transversal sin generar tensiones en dicha dirección. Asimismo, para tener en cuenta el efecto del jet de acero líquido entrante sobrecalentado sobre el frente de solidificación, se aumenta artificialmente la conductividad térmica del acero líquido, a partir de los datos del modelo CFD. Para el cálculo de las propiedades termomecánicas del acero se usa un modelo de microsegregación basado en la ecuación analítica de ClyneKruz desarrollada por Won y Thomas ${ }^{[4]}$. El modelo constitutivo utilizado para la modelización del creep está basado en el modelo elasto-visco-plástico III de Kozlowski ${ }^{[5]}$. Por otro lado, para evitar la generación de tensiones y deformaciones significativas en el líquido se ha considerado un creep muy

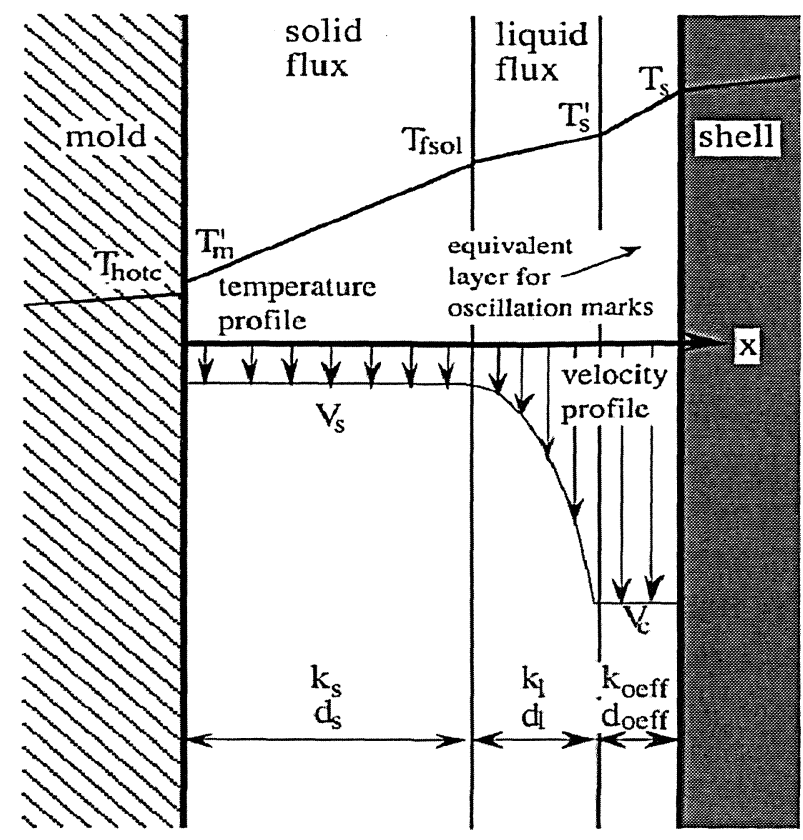

Figura 2. Perfiles de temperatura y velocidad en la región de fundentes.

Figure 2. Temperature and velocity profiles in the gap.

elevado para temperaturas superiores a la correspondiente al punto ZST (Zero Stress Temperature).

\section{RESULTADOS Y DISCUSIÓN}

El modelo global CFD ha sido validado parcialmente verificando el incremento de temperatura del agua de refrigeración con datos experimentales, resultando $5,8^{\circ} \mathrm{C}$ en el modelo CFD, y $6{ }^{\circ} \mathrm{C}$ en la colada real. En las siguientes gráficas se presenta la evolución de las variables involucradas en el modelo de transferencia de calor en la interfase molde-palanquilla, para el centro de la cara y el vértice, así como dos imágenes representativas de la simulación llevada a cabo mediante CFD.

En el vértice, el espesor de capa solidificada es mayor que en el centro de las caras, debido al efecto esquina. Por otro lado, para una posición angular dada en la sección de la palanquilla, se predice la altura a la que todo el fundente se solidifica $\left(d_{l}=0\right)$ y, por tanto, la región del molde por debajo de la cual hay potenciales problemas (irregularidades) en la transferencia de calor debido a la creación de huecos de aire en la interfase aceromolde. Debido igualmente al efecto vértice, el fundente solidifica antes en el vértice que en el centro de la cara (Fig. 3). Durante la solidificación del fundente la transferencia de calor va disminuyendo significativamente, ya que el espesor, $d_{s}$, debe aumentar ( $y$, por tanto, la resistencia térmica de 


\section{Cara}

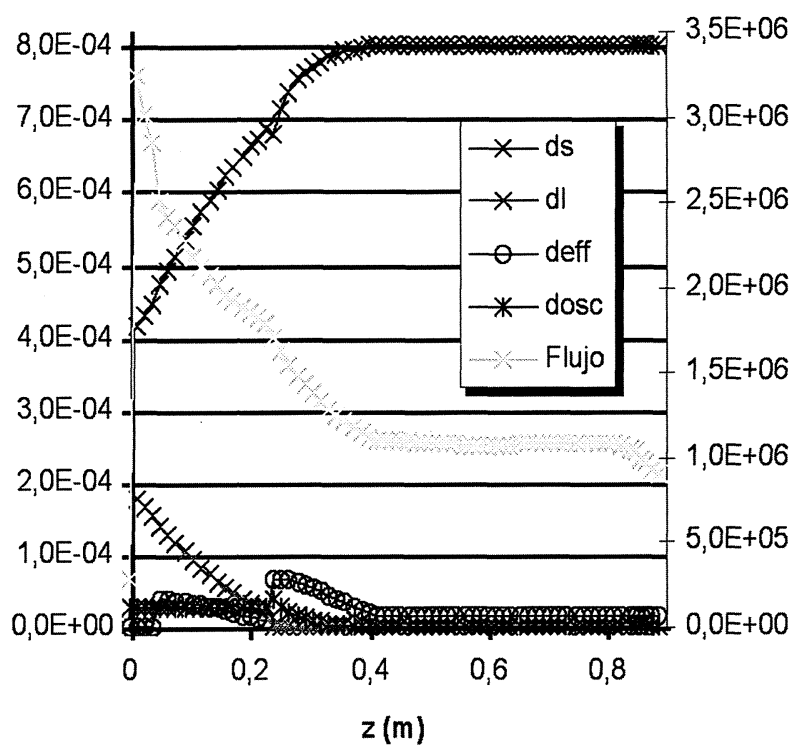

Vértice

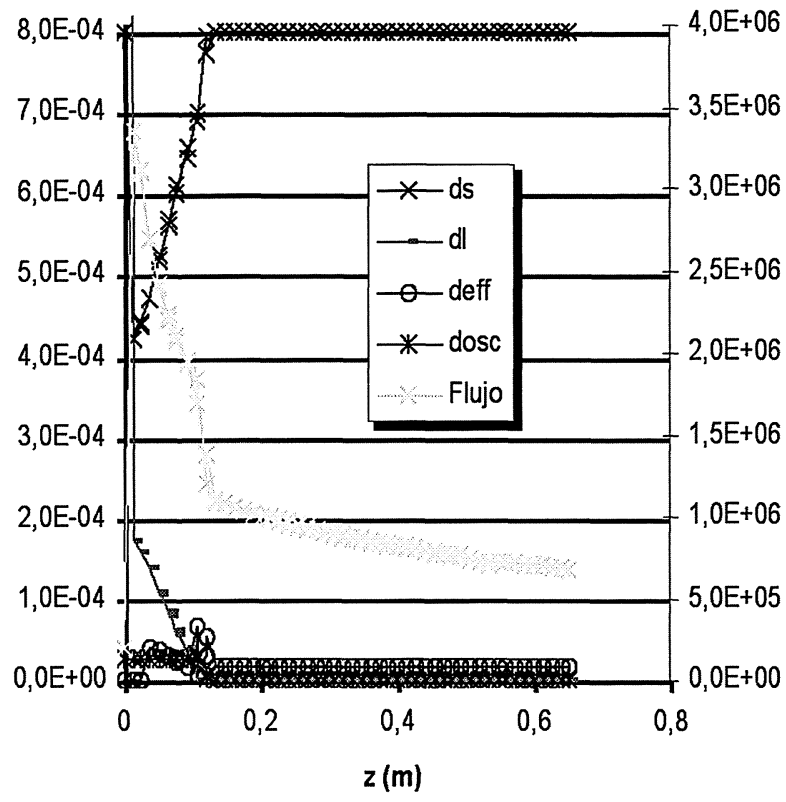

Figura 3. Flujos de calor y espesores de fundente en la interfase molde-acero.

Figure 3. Heat flux and flux thickness in the mould-steel interface.

los fundentes crece igualmente) para cumplir el balance de la ecuación (1), al estar multiplicado por una velocidad bastante inferior a la de colada. Asimismo, se refleja la influencia de efectos locales producidos por la entrada del acero, a través de la buza, en el avance de la capa solidificada. Se observa cómo la recirculación del acero (Fig. 4) produce un aumento de la transferencia de calor del acero recalentado hacia el frente de solidificación (es una zona de mayor velocidad y turbulencia del acero líquido), que implica un freno al avance del frente de solidificación. Así, es posible analizar la probabilidad de rotura de línea como consecuencia de un excesivo estrechamiento de la capa solidificada.

A continuación, en la imagen de la izquierda (Fig. 5) se muestra la deformada de la palanquilla aumentada por un factor de 10 a $285 \mathrm{~mm}$ de la salida del molde. Se observa cómo, en el vértice, la separación es mayor respecto a la referencia del molde; en parte, dicha forma es debida al efecto bisagra que ejerce el vértice al actuar la presión ferrostática sobre la cara, que tiende a abombarla, pero, por otro lado, también adopta dicha forma (aunque menos acusada) al resolver el caso sin presión ferrostática, debido a que el vértice se contrae más que la cara, al estar más frío.

En la imagen de la derecha se presenta la evolución de la temperatura y tensión en dirección paralela a la cara (S11) para el punto central de la su- perficie de la cara (punto $s$ ), y otro punto central a $10 \mathrm{~mm}$ del primero en el interior (punto $i$ ), en función de la distancia al menisco, $z$. La capa superficial se halla en tracción (suseptibilidad a grietas) hasta poco antes de la salida del molde, dado que, por un lado, actúa la presión ferrostática creciente y, por otro lado, al enfriarse más rápidamente que el interior (salvo la zona marcada por *), y por consiguiente, contraerse más rápido que las capas internas, a medida que éstas últimas resisten, se genera un esquema de tensiones de tracción superficial y compresión en el interior. Justo, antes de la salida del molde, se da una zona de ligero sobrecalentamiento superficial y se invierte la tendencia. Este sobrecalentamiento se explica si consideramos la transferencia de calor por conducción en 1D en la superficie (hipótesis válida en el centro de la cara):

$$
\rho c_{p}=\frac{d T}{d t}=k \frac{d^{2} T}{d x^{2}}-q_{\text {molde }}
$$

El signo de $d T / d t$ viene dado por el balance del miembro derecho: al bajar el flujo de calor al final del molde, $q_{\text {molde }}$ (Fig. 3) y mantenerse inicialmente $\lambda d^{2} T / d x^{2}, d T / d t$ cambia de signo; éste fenómeno ocurre aunque se siga extrayendo calor por el molde. Después, a la salida del molde, el flujo de calor aumenta bruscamente en la zona de sprays y el proceso vuelve a invertirse. 


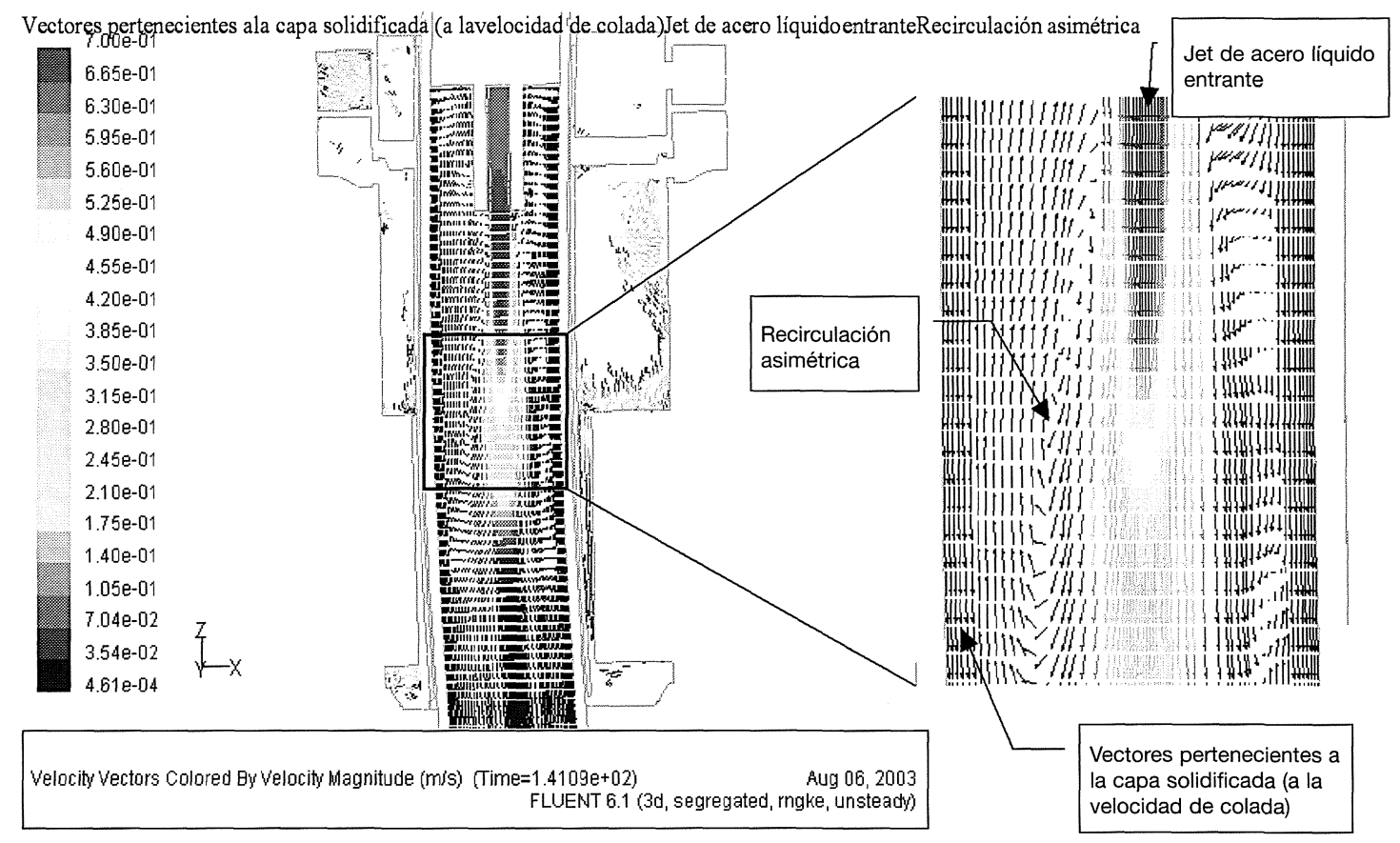

Figura 4. Vectores velocidad en el acero en un plano vertical.

Figure 4. Temperature and velocity profiles in the gap.
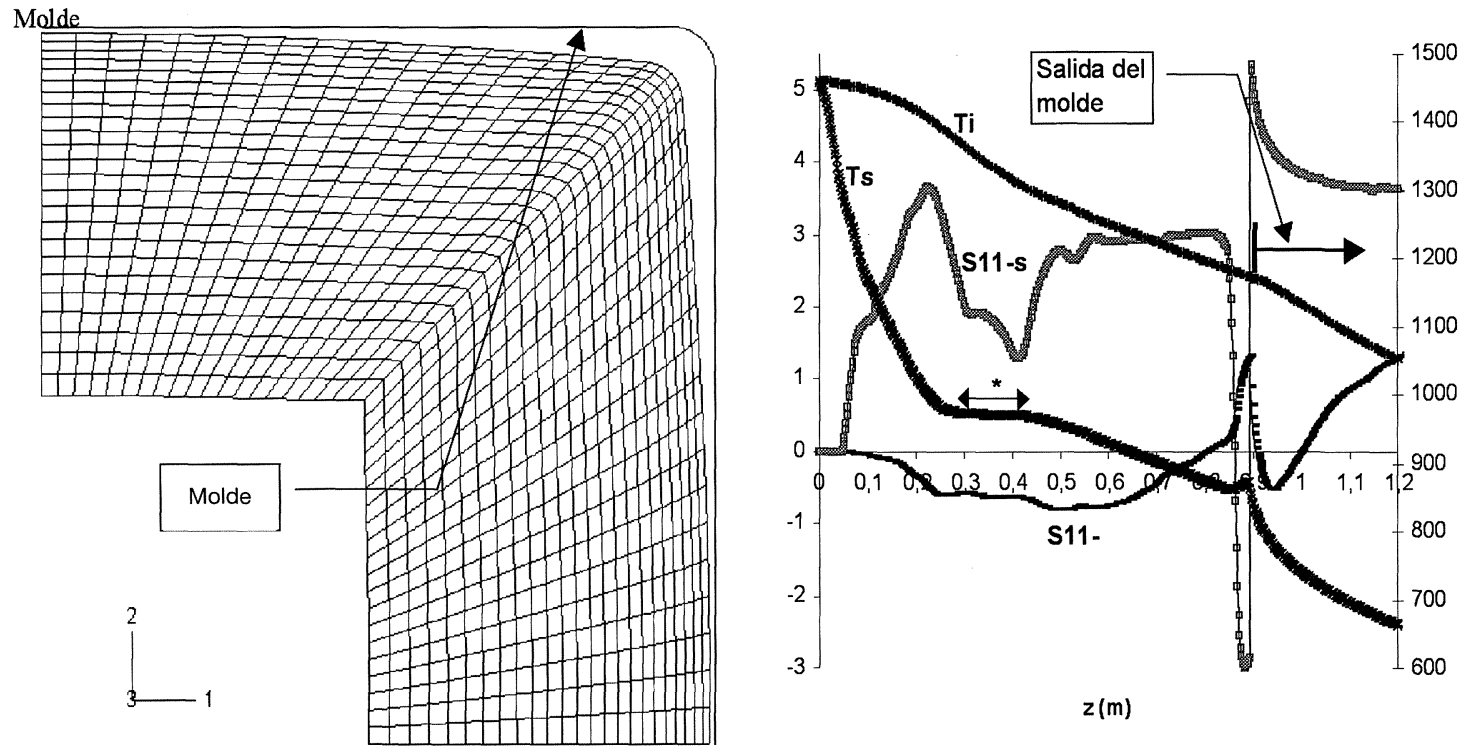

Figura 5. Deformada de la palanquilla y evolución de la temperatura y tensión en dirección 11.

Figure 5. Billet deformed shape and evolution of temperature and stress in 11 direction.

\section{CONCLUSIONES}

Se ha aplicado la metodología descrita a un modelo 3D de una colada continua real de palanquilla. Esta metodología permite resolver de forma precisa el flujo del acero líquido entrante, la solidificación del acero y de los fundentes y hace un tratamiento detallado de la transferencia de calor a través de éstos en la interfase acero-molde. También permite obtener tensiones y deformaciones de la capa solidificada de acero imponiendo en un modelo FEM 2D transversal los flujos obtenidos mediante CFD. 
Como datos de entrada a los modelos se requiere el consumo de fundentes, temperatura de entrada del acero líquido y el agua, y propiedades de los materiales.

En futuras simulaciones se tendrán en cuenta la conicidad del molde y se implementará un modelo de daño en el modelo FEM. Está previsto igualmente llevar a cabo estudios de sensibilidad de la colada respecto a variables como el consumo y propiedades de los fundentes, caudal de refrigeración, tipo de acero, tipo de buza (de salidas laterales), y velocidad de colada.

\section{REFERENCIAS}

[1] Y. Meng y B.G. Thomas, Metall. Mater. Trans. B (2003).

[2] J.K. Brimacombe, P.K. Agarwal, S. Hibbins, B. Prabhaker y L.A. BAPTISTA, Spray Cooling in Continuous Casting, Continuous Casting, Vol. 2, J.K. Brimacombe, Eds; ISS, Warrendale, PA, 1984, pp. 105-123.

[3] E. TakeuChi y J.K. BRimacombe, Metall. Trans. B 15 (1984) 493-509.

[4] Y.M. Won y B.G. Thomas, Metall. Mater. Trans. A 32 (2001) 1.755-1.767.

[5] P.F. Kozlowski, B.G. Thomas, J.A. Azi y H. Wang, Metall. Mater. Trans. A 23 (1992) 903-918. 